\title{
Effectiveness of whole-exome sequencing and costs of the traditional diagnostic trajectory in children with intellectual disability
}

\author{
Glen R. Monroe, MS ${ }^{1,2}$, Gerardus W. Frederix, PhD³, Sanne M.C. Savelberg, BS 1,2, Tamar I. de Vries, BS', \\ Karen J. Duran, BS ${ }^{1,2}$, Jasper J. van der Smagt, MD'1, Paulien A. Terhal, MD', \\ Peter M. van Hasselt, MD, PhD ${ }^{4}$, Hester Y. Kroes, MD, PhD ${ }^{1}$, Nanda M. Verhoeven-Duif, PhD ${ }^{1,2}$, \\ Isaäc J. Nijman, PhD ${ }^{1,2}$, Ellen C. Carbo, BS ${ }^{1,2}$, Koen L. van Gassen, PhD1, Nine V. Knoers, MD, PhD ${ }^{1,2}$, \\ Anke M. Hövels, PhD ${ }^{3}$, Mieke M. van Haelst, MD, PhD ${ }^{1}$, Gepke Visser, MD, PhD ${ }^{4}$ and \\ Gijs van Haaften, $\mathrm{PhD}^{1,2}$
}

\begin{abstract}
Purpose: This study investigated whole-exome sequencing (WES) yield in a subset of intellectually disabled patients referred to our clinical diagnostic center and calculated the total costs of these patients' diagnostic trajectory in order to evaluate early WES implementation.

Methods: We compared 17 patients' trio-WES yield with the retrospective costs of diagnostic procedures by comprehensively examining patient records and collecting resource use information for each patient, beginning with patient admittance and concluding with WES initiation. We calculated cost savings using scenario analyses to evaluate the costs replaced by WES when used as a first diagnostic tool.
\end{abstract}

Results: WES resulted in diagnostically useful outcomes in $29.4 \%$ of patients. The entire traditional diagnostic trajectory average cost was

\section{INTRODUCTION}

The advent of next-generation sequencing-first in the research environment and recently in diagnostic laboratories-provides a powerful tool to interrogate the exome, or even the entire genome, without a priori knowledge in pediatric patients with a disorder that has a suspected underlying genetic cause. ${ }^{1,2}$ The burden of genetic disease in child health is increasingly recognized, with an estimated $34 \%$ of inpatient children admissions having a clear genetic underlying cause, of which a significant and expanding proportion are identified as Mendelian disorders. ${ }^{3,4}$ Whole-exome sequencing (WES) enables examination of the coding part of the genome for variants in genes linked to Mendelian disorders. Trio-based WES of patients with suspected rare, monogenic disorders and their healthy parents has previously been demonstrated as a particularly effective method to find the causal variant in cases suspected to be caused by a de novo variant or due to autosomal recessive inheritance. WES gives value to parents by allowing timely interventions and
$\$ 16,409$ per patient, substantially higher than the $\$ 3,972$ trio-WES cost. WES resulted in average cost savings of $\$ 3,547$ for genetic and metabolic investigations in diagnosed patients and $\$ 1,727$ for genetic investigations in undiagnosed patients.

Conclusion: The increased causal variant detection yield by WES and the relatively high costs of the entire traditional diagnostic trajectory suggest that early implementation of WES is a relevant and costefficient option in patient diagnostics. This information is crucial for centers considering implementation of WES and serves as input for future value-based research into diagnostics.

Genet Med advance online publication 4 February 2016

Key Words: clinical management; cost analysis; intellectual disability; whole-exome sequencing

The first two authors and the last two authors contributed equally to this work.

${ }^{1}$ Department of Genetics, Utrecht University Medical Center, Utrecht, The Netherlands; ${ }^{2}$ Center for Molecular Medicine, Utrecht University Medical Center, Utrecht,

The Netherlands; ${ }^{3}$ Division of Pharmacoepidemiology and Clinical Pharmacology, Utrecht Institute of Pharmaceutical Sciences, Utrecht University, Utrecht, The Netherlands;

${ }^{4}$ Department of Metabolic Diseases, Wilhelmina Children’s Hospital, Utrecht, The Netherlands. Correspondence: Gerardus W. Frederix (G.W.J.Frederix@uu.nl) and Gijs van Haaften (G.vanHaaften@umcutrecht.nl) 
a "diagnostic odyssey," subjected to numerous hospitalizations, diagnostic tests, and procedures over the course of many yearspossibly with no diagnosis. In the Netherlands, these patients eventually end up in tertiary-level diagnostic facilities such as the STC.

The patients referred to the STC with intellectual disability seem highly suitable for a WES-based diagnostic approach because single-gene disorders account for at least one-quarter of intellectual-disability cases. ${ }^{11}$ The present study compared the effectiveness of WES with that of traditional diagnostic investigations. We provide an extensive overview of the costs of the traditional diagnostic trajectory in this patient group. We also examine the cost savings that early implementation of WES would enable by rendering various genetic or metabolic tests unnecessary. The findings of this research can be used as input in the discussion regarding the societal, individual, and monetary value of next-generation sequencing. ${ }^{12} \mathrm{~A}$ recent study assessed comprehensive costs of the traditional diagnostic pathway in a different group of patients, with complex pediatric neurological disorders. ${ }^{13}$ However, a clear determination of the diagnostic yield when actually performing WES compared with traditional diagnostic procedures and costs was not made. Reliable information on all resources used in the current clinical and diagnostic pathway is crucial for clinical diagnostic centers considering implementing WES as a diagnostic tool. In addition, this information could serve as input for future valuebased research for the diagnostic trajectory.

In this study, we applied patient-parent trio WES to 17 unsolved cases to identify the genetic cause of the patients' disorders and concurrently assessed the retrospective costs of diagnostic investigation for these patients using traditional methods. In $29.4 \%$ of the cases, WES analysis detected variants in genes recently discovered to cause Mendelian disorders or in genes mutated in patients with similar phenotypes. The estimated cost of WES is substantially lower than the mean cost $(\$ 16,409)$ of the traditional diagnostic trajectory for these patients. Early WES implementation would replace genetic and metabolic tests in patients who receive a diagnosis and replacement of genetic tests in patients who do not receive a diagnosis, resulting in cost savings of $\$ 3,547$ and $\$ 1,727$, respectively. This information suggests that WES should be considered a valid first tool in diagnostics in clinical diagnostic centers.

\section{Patient selection}

\section{MATERIALS AND METHODS}

Approximately 90 patients per year are seen at the STC in comprehensive one-day visits by a multidisciplinary team consisting of a clinical geneticist, a pediatric neurologist, a metabolic pediatrician, a physiotherapist, a psychologist, an ophthalmologist, a radiologist, and, on request, a psychiatrist and a dermatologist. From the 86 patients in the 2011 population, a subset of patients was selected that was suitable for WES based on the following criteria: the patients were born to healthy, unaffected parents; the parents were self-reported as nonconsanguineous; both parents could be contacted and were able to give consent; and the patient was undiagnosed at the time of the study. Seventeen patients were then randomly enrolled for inclusion in this study (Figure 1). All patients were intellectually disabled and as a cohort had wide and diverse phenotypes (Table 1). The parents of the patients signed informed consent for WES.

\section{Next-generation sequencing}

Parent-patient trio WES was performed with an emphasis on de novo variant detection analysis for the causal variants. DNA libraries for the 17 patients and their parents were prepared using Kapa Biosystems reagents (Kapa Biosystems, Wilmington, MA), enriched using Agilent Sureselect All Exon V5 (Agilent, Santa Clara, CA) and a custom pooling protocol (Supplementary Materials and Methods online), and sequenced on a HiSeq 2500 (Illumina, San Diego, CA). All trios were sequenced to a desired minimal internal quality standard of $75 \times$ mean bait coverage for the patient, $65 \times$ mean bait coverage for the parent, and the percentage target bases over $20 \times>85 \%$. Quality was often higher than this

Syvlia Tóth Center 2011 patient population

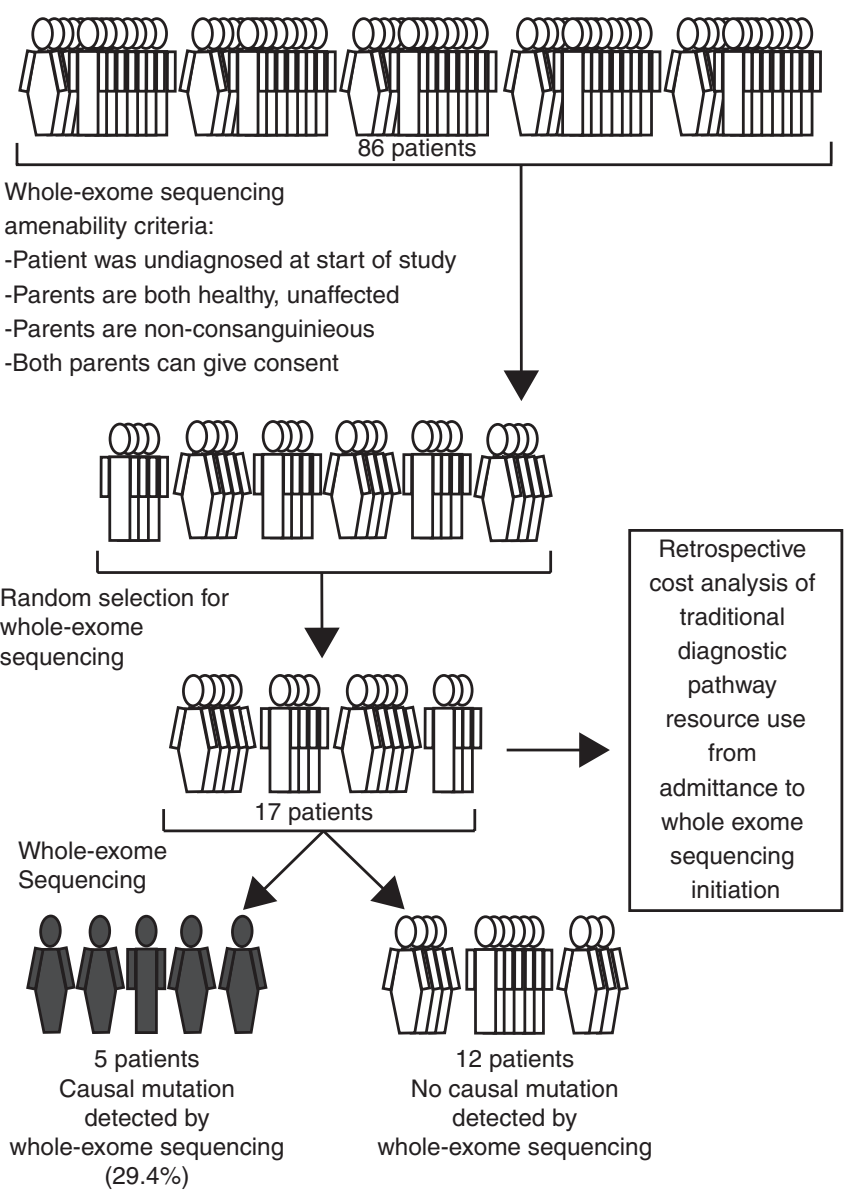

Figure 1 Study outline. Of the 86 patients of the Sylvia Tóth Center 2011 patient population, WES was performed in a 17-patient subset. Of these 17 patients, the causal variant was detected in 5 (29.4\%). Concurrently, a comprehensive retrospective cost analysis of the patients' individual traditional diagnostic pathway was performed. WES, whole-exome sequencing. 
Table 1 Patient information, length of diagnostic odyssey, and causal variant if identified

\begin{tabular}{|c|c|c|c|c|c|}
\hline Patient \# & Phenotype & Sex & $\begin{array}{l}\text { Age at } \\
\text { admittance } \\
\text { (years) }\end{array}$ & Causal variant & $\begin{array}{l}\text { Diagnostic } \\
\text { trajectory } \\
\text { duration } \\
\text { (years) }\end{array}$ \\
\hline 1 & Intellectual deficit, severe hypotonia, joint hypermobility, and obesity & Female & 1.1 & Not identified & 6.5 \\
\hline 2 & $\begin{array}{l}\text { Microcephaly, developmental delay, feeding problems, and severe } \\
\text { hypotonia }\end{array}$ & Female & 0.8 & $\begin{array}{l}\text { CTNNB1 NM_001904.3: } \\
\text { c.1925_1926delAG, } \\
\text { p.(Glu642Valfs*5)21 }\end{array}$ & 14.2 \\
\hline 4 & $\begin{array}{l}\text { Prenatal short stature (-3 SD), intellectual deficit, dysmorphic facial } \\
\text { features, obesity, radio ulnar synostosis, and hearing loss }\end{array}$ & Female & 2.2 & $\begin{array}{l}\text { ANKRD11 } \\
\text { NM_001256182.1: } \\
\text { c.3382_3383delGA, } \\
\text { p.(Asp1128GInfs*41)22 }\end{array}$ & 8.6 \\
\hline 5 & $\begin{array}{l}\text { Intellectual deficit, microcephaly, short stature, epilepsy, brain anomalies, } \\
\text { simian creases, and severe hypotonia }\end{array}$ & Male & 0.0 & Not identified & 4.4 \\
\hline 7 & $\begin{array}{l}\text { Macrocephaly (+2.5 SD), a prominent forehead with nevus flammeus, } \\
\text { psychomotor retardation, and seizures. Assymetry of the brain ventricles, } \\
\text { asymmetric lesions in periventricular white matter and basal ganglia, } \\
\text { polymicrogyria of perisylvian regions, cerebral atrophy and a relatively } \\
\text { small vermis cerebelli }\end{array}$ & Female & 0.8 & Not identified & 4.7 \\
\hline 8 & Severe syndromic intellectual disability & Female & 0.6 & $\begin{array}{l}\text { SMARCB1 } \\
\text { NM_O03073.3: } \\
\text { c. } 1113 C>\text { G } \\
\text { p.(Asn371Lys) }\end{array}$ & 5.8 \\
\hline 9 & Severe syndromic intellectual disability & Male & 0.0 & Not identified & 4.4 \\
\hline 10 & $\begin{array}{l}\text { Born preterm (GA 36) with an omphalocele, Apgar score of 2/7, } \\
\text { and development delayed from birth. Cyclic neutropenia and } \\
\text { trombopathy caused by a storage pool deficiency. Diminished fasting } \\
\text { tolerance resulting in hypoglycemia, disturbed gastric tract motility, } \\
\text { nephropcalcinosis and delayed myelinization. Muscle biopsy revealed a } \\
\text { diminished ATP production and diminished activity of multiple complexes } \\
\text { but no mitochondrial DNA abnormalities were detected }\end{array}$ & Male & 1.0 & Not identified & 7.7 \\
\hline 13 & Psychomotor delay, mainly as a result of spastic diplegia & Male & 2.6 & Not identified & 3.4 \\
\hline 14 & $\begin{array}{l}\text { Macrocephaly (+2.5 SD), hypertelorism, remarkable hyperlaxity of the } \\
\text { finger joints, and relatively small ears. Fused cervical vertebrae C2-C6, } \\
\text { bilateral total tarsal coalition, and psychomotor retardation. MRI of the } \\
\text { brain revealed a Chiari } 1 \text { malformation. Conductive hearing loss }\end{array}$ & Female & 11.8 & $\begin{array}{l}\text { CHD4 NM_001273.2: } \\
\text { c.3518G>T, } \\
\text { p.(Arg1173Leu) (variant } \\
\text { in candidate gene) }\end{array}$ & 3.7 \\
\hline 15 & $\begin{array}{l}\text { Intellectual deficit, short stature, macrocephaly, feeding problems, } \\
\text { disturbed sleeping pattern, mobility problems requiring wheelchair use }\end{array}$ & Female & 11.5 & Not identified & 3.9 \\
\hline 16 & $\begin{array}{l}\text { Mild intellectual disability with a verbal IQ of } 81 \text { and a performance IQ of } \\
56 \text {. Stocky build, brachydactyly (similar to his mother), a sandal gap, pes } \\
\text { planus, and mild joint laxity }\end{array}$ & Male & 7.6 & Not identified & 3.7 \\
\hline 17 & $\begin{array}{l}\text { Intellectual deficit, dysmorphic facial features, short stature (<-2.5 SD), } \\
\text { and trichotillomania }\end{array}$ & Female & 4.7 & Not identified & 3.9 \\
\hline
\end{tabular}

ATP, adenosine triphosphate; GA, gestational age; IQ, intelligence quotient; MRI, magnetic resonance imaging.

minimum requirement, with a mean coverage of $93 \times$ for the patients, $92 \times$ for the parents, and $90 \%$ for target bases over 20× (Supplementary Figure S1 online). Variant call files were imported into Cartagenia Bench Lab (https://cartagenia.com/) for additional variant interpretation, as well as into
Combined Annotation Dependent Depletion (http://cadd. gs.washington.edu/) for variant prioritization. ${ }^{14}$ Emphasis in analysis using Cartagenia was placed initially on an anticipated de novo inheritance of a deleterious variant, but autosomal recessive, $\mathrm{X}$-linked recessive, and compound heterozygote 
inheritance were subsequently investigated as well. Using the Cartagenia tool, de novo analysis was performed by filtering for Mendelian violations and prioritizing the variants using a classification tree by their presence in public population frequency databases, clinical variant databases, or our in-house data set (Supplementary Materials and Methods online). Variants were further prioritized by amino acid change or splicing effect, evolutionary conservation score (genomic evolutionary rate profiling; GERP), ${ }^{15}$ and predicted effect on protein function using prediction algorithms (Polyphen2, SIFT) ${ }^{16,17}$ and relevant literature. Variants were initially validated and segregation analysis performed by Sanger sequencing in a research setting and subsequently validated, along with segregation analysis, in the Genome Diagnostics section at the University Medical Centre (UMC) Utrecht. Primer information is available upon request.

\section{Retrospective cost analysis}

For the cost analysis, we set up a retrospective bottom-up cost of illness study from a hospital perspective. Data collection began for each patient at the first visit to the UMC Utrecht until initiation of WES.

All available resource use data were retrospectively collected from the hospital information systems and patient records. The data consisted of all health-care-professional visits, hospitalizations, imaging, genetic tests, metabolic investigations, and biochemical investigations performed at the UMC Utrecht. These collective investigations constitute the traditional diagnostic pathway. In addition, information about genetic tests performed at other genetics centers was collected through referral letters from general hospitals or other university medical centers. In addition, age at first visit and total length of diagnostic trajectory were recorded.

All individual units of care were then linked to their unit costs. Unit costs of resource use were derived from various sources. Reimbursement prices issued by the Dutch Healthcare Authority were used as individual unit costs for medical interventions, imaging and diagnostics, biochemical analysis, and surgeries. For inpatient days, health-care-professional visits, day admission, and blood products, national reference prices established by previous research were used. ${ }^{18}$ WES cost was estimated at $\$ 3,972(€ 3,600)$ per trio for ease of comparison with previous studies. ${ }^{13}$ This includes the costs of patient registration and blood draw, DNA isolation, sample preparation, exome enrichment, sequencing on an Illumina HiSeq 2500, interpretation, reporting of results, data storage, and infrastructure. All costs were indexed to 2014 levels using rates issued by the Dutch Healthcare Authority. Euros were converted to dollars using the 2 November 2015 official exchange rate of the European Central Bank $(€ 1=\$ 1.1032)$.

\section{Cost scenario analyses}

To appraise the impact of introducing WES early in the patient diagnostic trajectory, we created additional cost scenario analyses evaluating which procedures would be unnecessary if WES was performed initially. After WES was performed, we divided the patients into two groups-diagnosed and undiagnosedand for each group we calculated the total amount of costs that could potentially be saved by a WES-first approach in diagnostics. For the diagnosed patients, WES would replace all genetic costs (except for array comparative genomic hybridization (aCGH) and single-nucleotide polymorphism (SNP) arrays) and all metabolic assessments. For undiagnosed patients, WES would replace all genetic costs except aCGH/SNP array assessments. For both groups, aCGH/SNP arrays will continue to be performed to exclude copy-number variants. We created a third scenario for these two groups assuming that WES will result in a cost reduction of $50 \%$ for health-care visits, imaging, biochemical investigations, and patient day admission. Having WES results can reduce health-care visits by eliminating the need for additional visits to discuss negative genetic or metabolic results, to avoid redundant imaging or biochemical investigations, and to spend less time in patient day admission.

\section{WES yield}

\section{RESULTS}

We prioritized de novo variants, as the selected patients were born of healthy, nonconsanguineous parents. After filtering for only exonic and essential splice-site variants, a total of 38 de novo variants remained. We validated 32 of the variants by Sanger sequencing, resulting in a validation rate of $84 \%$, with a mean value of 1.88 de novo variants for each patient and a range of 0 to 5 de novo variants per patient in the exome. A full list of de novo variants for each individual patient is provided in Supplementary Table S1 online. The value of 1.88 validated de novo variants per patient in our study is consistent with the 1.71-1.98 validated de novo variants per patient previously reported in larger intellectual-disability cohorts, thereby validating our sequencing and analysis pipeline. ${ }^{19,20}$ Autosomal recessive, X-linked recessive, and compound heterozygous inheritance models were also investigated, but no variants that could explain the patients' phenotype were detected. The lack of homozygous autosomal recessive causal variants was in accordance with our initial selection of only patients whose parents were nonconsanguineous.

We considered a variant diagnostic only if other patients with a clear phenotypic overlap had been described and harbored variants with comparable predicted damaging effect in the same gene. Diagnostic variants were found in 5 of the 17 patients, resulting in a yield in known or candidate Mendelian disease genes in this cohort of 29.4\%. Variants in CTNNB1 (catenin (cadherin-associated protein), beta $1,88 \mathrm{kDa}), A N K R D 11$ (ankyrin repeat domain 11), ADNP (activity-dependent neuroprotector homeobox), SMARCB1 (SWI/SNF related, matrix associated, actin dependent regulator of chromatin, subfamily b, member 1), and CHD4 (chromodomain helicase DNA binding protein 4) were causal for five patients' phenotype (Table 1). Patients 2 and 4 further delineated the phenotype of the previously identified CTNNB1 haploinsufficiency syndrome and KBG syndrome, respectively. ${ }^{21,22}$ Variants identified in $A D N P$ (patient 6) and SMARCB1 (patient 8) provided a diagnosis of 
ADNP syndrome and Coffin-Siris syndrome. In addition, for patient 14 the CHD4 variant has a strong likelihood of being causal given that similar missense variants within $\mathrm{CHD} 4$ were previously reported in the DDDUK study (https://decipher. sanger.ac.uk), with a strong similarity between our patient's phenotype and the reported phenotype. ${ }^{23-25}$ Further functional work and similar patients with variants in $\mathrm{CHD} 4$ are required to establish causality. A clinical geneticist returned the WES results to the patients and parents.

\section{Retrospective cost analysis}

We performed a retrospective cost analysis for all 17 patients involved in this study. The complete data set is available on request. Patients first visited the UMC Utrecht at a median of 1.1 years of age, and an average of 3.0 years of age. The average duration of the diagnostic trajectory was 6.6 years (Table 1). For our cost analysis we focused on the diagnostic trajectory costs: health-care visits, imaging, genetics, metabolic measurements, biochemical investigations, and patient day admission (Table 2 and Supplementary Table S2 online). Costs for WES were not included. On average, patients had 61 visits with health-care professionals, with the majority of these being visits to see a medical professional (for instance, a medical specialist, nurse, or physiotherapist), with a mean cost of $\$ 3,012$ (median: $\$ 2,144$; range: \$435-\$9,844). Patients underwent imaging, with the majority being X-ray and magnetic resonance imaging, an average of 16 times, with mean costs of $\$ 1,439$ (median: $\$ 771$; range: $\$ 0-\$ 7,981)$. An average of seven genetic tests per patient were performed, with a mean cost of $\$ 6,588$ (median: $\$ 5,745$; range: $\$ 2,183-\$ 20,476)$. The majority of these genetic tests comprise single-gene tests (65\%). A mean of 1.5 aCGH/SNP array tests (as technology improved) were performed per patient (median: 1 ; range $1-3$ ), at a mean cost of $\$ 1,361$ (median: $\$ 890$; range: $\$ 890-\$ 2,670)$. An average of six metabolic tests were performed per patient, with a mean cost of $\$ 2,818$ (median: $\$ 2,777$; range: $\$ 2,204-\$ 4,343$ ) and mean biochemical investigation costs of $\$ 2,034$ (median: $\$ 355$; range: $\$ 140-\$ 15,457$ ). On average, patients were admitted during the day four times, costing $\$ 517$ (median: $\$ 309$; range: $\$ 0-\$ 3,243$ ). The mean total costs per patient were $\$ 16,409$, median costs were $\$ 14,153$, ranging from $\$ 6,343$ to $\$ 47,841$ for the total diagnostic trajectory.

Table 2 Overview of mean/median number and costs of traditional diagnostic trajectory

\begin{tabular}{lcccc} 
& $\begin{array}{c}\text { No. } \\
\text { (median) }\end{array}$ & $\begin{array}{c}\text { No. } \\
\text { (mean) }\end{array}$ & $\begin{array}{c}\text { Costs } \\
\text { (median) }\end{array}$ & $\begin{array}{c}\text { Costs } \\
\text { (mean) }\end{array}$ \\
\hline Health-care visits & 38 & 61 & 2,144 & 3,012 \\
Imaging & 8 & 16 & 771 & 1,439 \\
\hline Genetics & 6 & 7 & 5,745 & 6,588 \\
\hline Metabolics & 6 & 6 & 2,777 & 2,818 \\
\hline Biochemical investigations & 5 & 28 & 355 & 2,034 \\
Day admission & 2 & 4 & 309 & 517 \\
\hline Total & & & 14,153 & 16,409 \\
\hline
\end{tabular}

Values are given in USD.
The costs per patient are shown in Figure 2. On average, the genetic costs were $42 \%$ of the mean total diagnostic trajectory costs, ranging from 11 to $76 \%$. We were able to diagnose patients 2, 4, 6, 8, and 14 following WES. The total diagnostic trajectory until WES for diagnosed patients took, respectively, $14.2,8.6,3.3,5.8$, and 3.7 years. The total traditional diagnostic trajectory costs for these patients were, respectively, $\$ 23,173$, $\$ 14,187, \$ 6,343, \$ 5,893$, and $\$ 9,934$.

\section{Cost scenario analyses}

In three hypothetical scenarios, we evaluated the impact of WES on cost savings with the assumption that WES would render certain diagnostic investigations unnecessary. If WES was performed as a first diagnostic approach for patients in our group who ultimately received a genetic diagnosis, cost savings of genetic testing and metabolic testing would average $\$ 4,986$ (median: $\$ 5,342$; range: $\$ 0-\$ 10,684$ ) and $\$ 2,533$ (median: $\$ 2,446$; range: $\$ 2,204-\$ 2,866$ ), respectively. For patients who did not receive a diagnosis following WES but for whom WES would replace genetic testing, savings would average $\$ 5,699$ (median: $\$ 4,854$; range: $\$ 890-\$ 18,696$ ).

In the final scenario, we assumed that WES would result in a $50 \%$ reduction of number and cost in the categories of health-care visits, imaging, biochemical tests, and patient day admission. For the diagnosed patients we calculated mean total savings of $\$ 1,660$ and for the undiagnosed patients $\$ 4,269$.

\section{DISCUSSION}

Implementation of WES in clinical practice is at a turning point. The application of WES in diagnostics has transformed the clinic and allowed massive interrogation of the entire coding region of

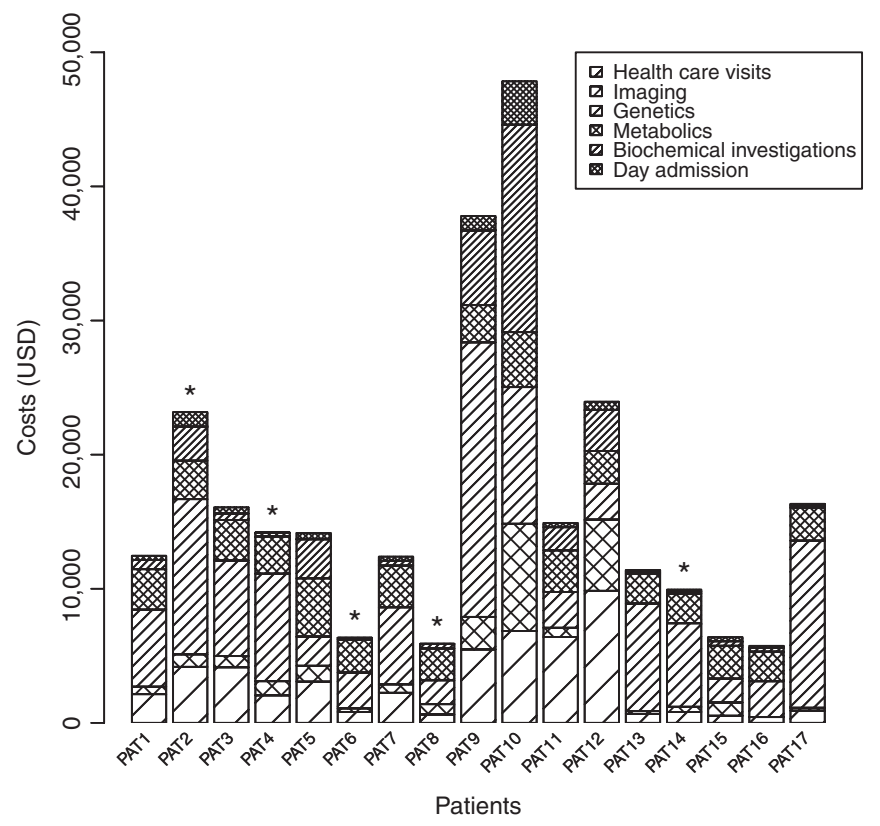

Figure 2 Overview of the costs of the traditional diagnostic trajectory per patient. The cost of patient-parents trio WES is not included. *Patients in whom the causal variant was identified. WES, whole-exome sequencing. 
the genome. The diagnostic yield has been proven in numerous studies, and the cost continues to fall. However, information is lacking on the prospective yield using WES compared with the cost of patient diagnostics retrospectively using the traditional diagnostic pathway. An initial study focused on retrospective cost analysis of patient resource use in a cohort of neurological patients and the potential application of WES. ${ }^{13}$ The current study not only links the costs of patient diagnostics using traditional means but also reveals the higher yield and lower costs when actually implementing WES in a heterogeneous, difficultto-diagnose patient group. This knowledge is essential for clinical diagnostic centers considering WES implementation so that the cost for increased diagnostic yield can be ascertained and considered if WES is advantageous.

The yield from this study further confirms that WES is useful in the clinic. Current single-gene disorder analyses or chromosomal microarray analyses have a diagnostic rate of $\sim 13-14 \%$ in these patient populations, emphasizing the need for new molecular technologies. ${ }^{26,27}$ Several previous reports using a trio-based WES strategy in large, specific patient populations reported diagnostic yields of $\sim 25 \%$, with some smaller studies reporting yields of up to $45-55 \% .{ }^{7,20,28-30}$ The yield in this study is comparable to the $25 \%$ from these large patient population yields, and it demonstrates the power of WES to identify the genetic cause of disease in cohorts of intellectually disabled patients. Recent studies have reported a diagnostic yield of 37\% using trio analysis via the inclusion of novel genes for which a variant was observed in only one patient. ${ }^{6}$ Our study's yield does not include variants in entirely novel genes without the support of additional patients with similar phenotypes or functional work to establish causality, as previous reports have demonstrated that "overinterpretation" of the exome can lead to false positives. ${ }^{31}$ We expect that future essential patient datasharing efforts and functional work will ultimately result in a higher yield for this patient cohort. Of note, the four previously known Mendelian genes that harbored diagnostic variants have only been known to be causal for these specific syndromes for less than 4 years, indicating the rapid progress in human genetics in the past few years. When this study was initiated, the underlying pathogenic mechanism for KBG (defects in $A N K R D 11)$ was still being elucidated and a diagnostic test had not yet been developed. Three of the genes-ADNP, CTNNB1, and SMARCB1-have only recently been associated with the respective syndromes. WES clearly has an advantage over traditional single-gene analysis in situations when a specific molecular test does not exist or for syndromes for which the genetic cause has only recently been discovered. For the cases that are still unsolved, the WES results can be reanalyzed periodically to determine whether a detected de novo variant can be linked to new discoveries.

Our study confirms the long traditional diagnostic trajectory and the high costs for traditional diagnostic testing in this patient population. We found a mean cost of $\$ 16,409$ per patient that is comparable to other total costs recently published, albeit for a different patient group. ${ }^{13}$ The largest proportion of costs
(42\%) was related to previously performed gene tests. A previous study examining WES yield in a cohort of 12 patients also estimated the high amount of resources used, with a single patient's laboratory investigations costing $\$ 22,000 .^{32}$ High prices for additional diagnoses were also reported by Shashi et al. ${ }^{33}$ to be $\$ 25,000$ per diagnosis if no diagnosis was obtained after a first visit. Soden et al..$^{34}$ recently estimated that negative diagnostic tests for a group of neurodevelopmental disorder patients cost $\$ 19,100$. In our study, patient 10 thus far has accrued a total of $\$ 47,841$ in costs and still requires a diagnosis. For these patients, whole-genome sequencing is the next step in the diagnostic investigation to fully interrogate the genome.

Utilizing a WES-first approach would have immediate cost savings for diagnosed as well as undiagnosed patients. With a WES cost of $\$ 3,972$ compared with a mean cost of $\$ 4,986$ for genetic tests and $\$ 2,533$ for metabolic tests, using WES would directly save, on average, $\$ 3,547$ per patient who receives a diagnosis and a mean savings of $\$ 1,727$ for patients who do not receive a diagnosis using WES.

Other savings may also be realized. Before WES was introduced, the differential diagnostic workup required additional investigations (e.g., cardiac or renal ultrasounds; skeletal $\mathrm{X}$-rays). If no such anomalies are reported in a genetic condition that has been diagnosed by WES, then there is no need to perform these additional investigations. These additional savings demonstrate not only that obvious genetic or metabolic tests can become redundant if WES is the first diagnostic approach but also that a proportion of other patient procedures could be omitted.

This research demonstrates that implementing WES as a first diagnostic tool for patients with intellectual disability, even in a tertiary center population, could reduce health-care costs because WES could replace a large number of genetic and metabolic investigations. Costs of WES are markedly lower than the average total traditional diagnostic trajectory costs; indeed, the cost of trio WES is already less than that of genetic investigations in all the patients in our study $(\$ 3,972$ compared with $\$ 6,588$ ). Minimally, WES would avoid genetic and metabolic tests in patients who receive a diagnosis and genetic tests in patients who do not receive a diagnosis. An additional proportion of savings would be realized, at a proportion that will vary depending on which auxiliary procedures or costs the use of WES will partially replace. Importantly, these scenarios require an initial critical stratification by a physician specialized in diagnosing patients with intellectual disability. Such a physician is able to recognize patients who have a clear clinical presentation suggesting a known underlying genetic cause that is not detectable by WES (e.g., fragile $\mathrm{X}$, trinucleotide repeat, or methylation disorders) and for whom WES would not be beneficial and a waste of resources. This indicates that WES should be considered first for the majority of cases in which a genetic condition is strongly suspected.

The current study has several limitations. Because we evaluated this procedure as a pilot project, the randomly selected sample size of 17 patients is limited. Definite conclusions 
regarding where WES should be implemented in the diagnostic pipeline cannot be drawn on the basis of such small numbers. However, the diagnostic yield was consistent with other studies, suggesting that this yield range is what can be expected if the study is expanded to include more patients. Moreover, the small number enabled us to disentangle individual diagnostic odysseys and their economic consequences.

The cost analysis study was as comprehensive as possible, but some additional costs may exist. Only direct medical costs were taken into account in this study, and resource use collection (except genetic tests) was restricted to the UMC Utrecht.

Also, if WES were applied earlier to our patient group, there would be a clear, quantifiable cost savings of genetic and metabolic tests in patients who receive a diagnosis and savings of genetic tests in patients who do not receive a diagnosis. The reduction of these costs represents the minimum savings that would be realized with earlier WES introduction. Additionally, the contribution of the auxiliary proportion of savings in the categories of health-care visits, imaging, biochemical investigations, and day admission is debatable. For instance, the $50 \%$ cost reduction that we assume may be lower for undiagnosed patients whom further diagnostic investigations are necessary. Indeed, investigations, metabolic or otherwise, can aid in the interpretation of WES results or elucidating the disease pathogenesis. ${ }^{35,36}$ It is important for future analyses to consider these additional savings and correctly categorize diagnostic procedures that can be reduced by WES at the time of care to enable precise estimates of cost savings.

In conclusion, this study links the increase in diagnostically useful findings enabled by WES in intellectually disabled patients with the costs of traditional diagnostic investigations. The increase in causal variant detection and speed of diagnosis and the lower cost of WES compared with traditional diagnostic investigations in this patient population suggest that WES should be implemented early in clinical diagnostic centers with similar patient populations.

\section{SUPPLEMENTARY MATERIAL}

Supplementary material is linked to the online version of the paper at http://www.nature.com/gim.

\section{ACKNOWLEDGMENTS}

We are grateful to the patients and their parents for their participation in this study. This study makes use of data generated by the DECIPHER community. A full list of centers that contributed to the generation of the data is available at http://decipher.sanger. ac.uk and via e-mail from decipher@sanger.ac.uk. Funding for the DECIPHER project was provided by the Wellcome Trust.

\section{DISCLOSURE}

The authors declare no conflict of interest.

\section{REFERENCES}

1. Ng SB, Buckingham KJ, Lee $C$, et al. Exome sequencing identifies the cause of a mendelian disorder. Nat Genet 2010;42:30-35.
2. Choi M, Scholl UI, Ji W, et al. Genetic diagnosis by whole exome capture and massively parallel DNA sequencing. Proc Natl Acad Sci USA 2009;106: 19096-19101.

3. McCandless SE, Brunger JW, Cassidy SB. The burden of genetic disease on inpatient care in a children's hospital. Am J Hum Genet 2004;74:121-127.

4. Hall JG, Powers EK, Mcllvaine RT, Ean VH. The frequency and financial burden of genetic disease in a pediatric hospital. Am J Med Genet 1978; 1 417-436.

5. Dixon-Salazar TJ, Silhavy JL, Udpa N, et al. Exome sequencing can improve diagnosis and alter patient management. Sci Trans/ Med 2012;4:138ra78.

6. Farwell KD, Shahmirzadi L, El-Khechen D, et al. Enhanced utility of familycentered diagnostic exome sequencing with inheritance model-based analysis: results from 500 unselected families with undiagnosed genetic conditions. Genet Med 2015;17:578-586.

7. Lee $H$, Deignan JL, Dorrani $N$, et al. Clinical exome sequencing for genetic identification of rare Mendelian disorders. JAMA 2014;312:1880-1887.

8. Srivastava S, Cohen JS, Vernon $\mathrm{H}$, et al. Clinical whole exome sequencing in child neurology practice. Ann Neurol 2014;76:473-483.

9. Iglesias A, Anyane-Yeboa K, Wynn J, et al. The usefulness of whole-exome sequencing in routine clinical practice. Genet Med 2014;16:922-931.

10. Engbers HM, Berger $R$, van Hasselt $P$, et al. Yield of additional metabolic studies in neurodevelopmental disorders. Ann Neurol 2008;64:212-217.

11. Strømme P. Aetiology in severe and mild mental retardation: a population-based study of Norwegian children. Dev Med Child Neurol 2000;42:76-86.

12. Phillips KA, Ann Sakowski J, Trosman J, Douglas MP, Liang SY, Neumann P. The economic value of personalized medicine tests: what we know and what we need to know. Genet Med 2014;16:251-257.

13. van Nimwegen KJ, Schieving JH, Willemsen MA, et al. The diagnostic pathway in complex paediatric neurology: a cost analysis. Eur J Paediatr Neurol 2015:19:233-239.

14. Kircher M, Witten DM, Jain P, O'Roak BJ, Cooper GM, Shendure J. A general framework for estimating the relative pathogenicity of human genetic variants. Nat Genet 2014;46:310-315.

15. Cooper GM, Stone EA, Asimenos G, Green ED, Batzoglou S, Sidow A; NISC Comparative Sequencing Program. Distribution and intensity of constraint in mammalian genomic sequence. Genome Res 2005;15:901-913.

16. Adzhubei IA, Schmidt S, Peshkin L, et al. A method and server for predicting damaging missense mutations. Nat Methods 2010;7:248-249.

17. Kumar P, Henikoff S, Ng PC. Predicting the effects of coding non-synonymous variants on protein function using the SIFT algorithm. Nat Protoc 2009;4: 1073-1081.

18. Tan SS, Bouwmans CA, Rutten FF, Hakkaart-van Roijen L. Update of the Dutch manual for costing in economic evaluations. Int J Technol Assess Health Care 2012;28:152-158.

19. Hamdan FF, Srour M, Capo-Chichi JM, et al. De novo mutations in moderate or severe intellectual disability. PLoS Genet 2014;10:e1004772.

20. Rauch A, Wieczorek D, Graf E, et al. Range of genetic mutations associated with severe non-syndromic sporadic intellectual disability: an exome sequencing study. Lancet 2012;380:1674-1682.

21. Kuechler $A$, Willemsen $M H$, Albrecht $B$, et al. De novo mutations in beta-catenin (CTNNB1) appear to be a frequent cause of intellectual disability: expanding the mutational and clinical spectrum. Hum Genet 2015;134:97-109.

22. Ockeloen $\mathrm{CW}$, Willemsen $\mathrm{MH}$, de Munnik S, et al. Further delineation of the KBG syndrome phenotype caused by ANKRD11 aberrations. Eur J Hum Genet 2015;23:1176-1185.

23. Bragin E, Chatzimichali EA, Wright CF, et al. DECIPHER: database for the interpretation of phenotype-linked plausibly pathogenic sequence and copy-number variation. Nucleic Acids Res 2014;42(Database issue): D993-D1000

24. Deciphering Developmental Disorders Study. Large-scale discovery of novel genetic causes of developmental disorders. Nature 2015;519:223-228.

25. Firth HV, Richards SM, Bevan AP, et al. DECIPHER: Database of Chromosomal Imbalance and Phenotype in Humans Using Ensembl Resources. Am J Hum Genet 2009;84:524-533.

26. Henderson LB, Applegate CD, Wohler E, Sheridan MB, Hoover-Fong J, Batista DA. The impact of chromosomal microarray on clinical management: a retrospective analysis. Genet Med 2014;16:657-664.

27. Hochstenbach R, van Binsbergen E, Engelen J, et al. Array analysis and karyotyping: workflow consequences based on a retrospective study of 36,325 patients with idiopathic developmental delay in the Netherlands. Eur J Med Genet 2009:52:161-169. 
28. de Ligt J, Willemsen $\mathrm{MH}$, van Bon BW, et al. Diagnostic exome sequencing in persons with severe intellectual disability. N Engl J Med 2012;367: 1921-1929.

29. Wright CF, Fitzgerald TW, Jones WD, et al.; DDD study. Genetic diagnosis of developmental disorders in the DDD study: a scalable analysis of genome-wide research data. Lancet 2015;385:1305-1314.

30. Yang Y, Muzny DM, Xia F, et al. Molecular findings among patients referred for clinical whole-exome sequencing. JAMA 2014;312:1870-1879.

31. Atwal PS, Brennan ML, Cox R, et al. Clinical whole-exome sequencing: are we there yet? Genet Med 2014;16:717-719.

32. Need AC, Shashi V, Hitomi Y, et al. Clinical application of exome sequencing in undiagnosed genetic conditions. J Med Genet 2012;49:353-361.
33. Shashi V, McConkie-Rosell A, Rosell B, et al. The utility of the traditional medical genetics diagnostic evaluation in the context of next-generation sequencing for undiagnosed genetic disorders. Genet Med 2014;16:176-182.

34. Soden SE, Saunders CJ, Willig LK, et al. Effectiveness of exome and genome sequencing guided by acuity of illness for diagnosis of neurodevelopmental disorders. Sci Trans/ Med 2014;6:265ra168.

35. Campeau PM, Kasperaviciute D, Lu JT, et al. The genetic basis of DOORS syndrome: an exome-sequencing study. Lancet Neurol 2014;13:44-58.

36. Patton MA, Krywawych S, Winter RM, Brenton DP, Baraitser M. DOOR syndrome (deafness, onycho-osteodystrophy, and mental retardation): elevated plasma and urinary 2-oxoglutarate in three unrelated patients. Am J Med Genet 1987;26:207-215. 